\title{
Prevention and Control Strategies for Coronavirus Disease-2019 in a Tertiary Hospital in the Middle East of China
}

This article was published in the following Dove Press journal: Risk Management and Healthcare Policy

\section{Qingfang Kong \\ Li Yan}

Department of Infection Prevention and Control, Zhongda Hospital Affiliated to Southeast University, Nanjing 210009,

People's Republic of China
Correspondence: Qingfang Kong Zhongda Hospital Affiliated to Southeast University, No. 87 Dingjiaqiao, Nanjing, People's Republic of China

Emailnjkqf@I63.com
Background: Coronavirus disease-2019 (COVID-19) is a new infectious disease. The COVID-19 outbreak has quickly spread globally and now is having remarkable effects on public health and national economies.

Purpose: Effective measures for prevention and control of nosocomial infection are urgently needed to control COVID-19 infection among medical staff.

Methods: Herein, we described COVID-19 prevention and control strategies that have been implemented in a tertiary hospital in the middle east of China. Hospital layout was adjusted to prevent and control transmission. Other procedures were then implemented to prevent the spread of the virus, including specialized training and evaluation of medical staff, pre-testing and triage, measures to promote early identification of patients, environmental cleaning and disinfection, new medical waste processing procedures, new patient management procedures, timely disinfection of potential sources of nosocomial infection, and strict protective measures.

Results: Since the new measures were implemented, to date there have only been three new confirmed cases of COVID-19 infection, no cases of medical staff infection, and no COVID19-related deaths at the hospital.

Conclusion: The current report provides a protocol that could be implemented in medical departments in similar cities worldwide.

Keywords: COVID-19, health-associated infection, infection management, SARS-CoV-2

\section{Introduction}

The virus that causes coronavirus disease-2019 (COVID-19) has been officially named severe acute respiratory syndrome coronavirus 2 (SARS-CoV-2) by the World Health Organization (WHO). ${ }^{1}$ Since the first COVID-19 patient was reported in Wuhan in December 2019, there have been 78,630 confirmed cases reported in China at 17:00 on 27 February 2020, of which 65,596 (83.4\%) were patients from Hubei Province. Cases have been transported between Chinese cities, as well as internationally. $^{2}$ There were 631 confirmed cases in Jiangsu Province, of which 93 (14.7\%) were from Nanjing, the capital of Jiangsu Providence.

Though COVID-19 was classified as a class B infectious disease by the infectious disease prevention and control, the prevention and control measures for class A infectious disease were recommended in China on 20 January $2020 .^{3}$ Up to 17 February 2020, more than 3000 medical staff in China have been infected with SARS-CoV-2. Unfortunately, more than 10 doctors and nurses have died from 
COVID-19 after contracting it during the course of diagnosing and treating others. Although SARS-CoV-2 infection is effectively controlled in Jiangsu, 13, 415 confirmed cases and 120 deaths related to COVID-19 in mainland China, outside Hubei province- the epicentre of the outbreak-had been reported as of 18 March $2020 .^{4}$ As of 28 May 2020, the COVID-19 pandemic has precipitated a global crisis, with a total of 5,593,631 cases and 353,334 confirmed deaths globally caused by COVID $-19.5^{5}$ The COVID-19 outbreak has rapidly spread globally, and this has had remarkable effects on public health and national economies..$^{6-8}$ The global epidemic is still at a key stage with regard to prevention and control, particularly with respect to preventing the importation of infected patients from overseas, and preventing associated spread of infection in the event that such patients are diagnosed in China. Accordingly, there is an urgent need to develop effective strategies to prevent and control the spread of COVID-19.

\section{Patients and Methods}

\section{Patients}

Between 21 January 2020 and 28 May 2020 more than 1860 patients with fever visited our hospital, which is one of the designated hospitals for suspected cases of COVID19 in Jiangsu Province, China. The study was approved by the Medical Ethics Committee of Zhongda Hospital affiliated to Southeast University. All participants provided written informed consent prior to participation.

\section{COVID-I 9 Prevention and Control at the Hospital}

Given that proper infection prevention and control measures and good hygiene practices are essential to prevent spread of COVID-19 and protect both patients and the health-care workers, ${ }^{9}$ the hospital implemented the following measures in line with our actual situation and requirements:

1. Establishment of a central leading organizational structure for prevention and control of COVID-19 (Figure 1). The hospital director, hospital secretary, and the chief commander of COVID-19 prevention and control command are directly responsible for coordinating the deployment of epidemic prevention and control measures. There are three vice-director group leaders, and one is in charge of the medical department, another the infection management department, and another the nursing department. In addition, the head of each department directly manages 15 members under these three departments. This tiered system of responsibility is conducive to improving work efficiency.

2. Adjustment of the layout of clinical departments. Based on the requirements of the hospital, the overall layout was adjusted and new isolation wards and coronavirus screening sampling points were established. In addition, a series of relatively independent areas were established including a fever clinic, outpatient and emergency pre-examination triage area, emergency room, computed tomography room, and pediatric emergency room. The isolation ward consists of three zones and two "passages". The three zones are a contaminated zone, a potentially contaminated zone, and a clean zone. One of the two passages is reserved for exclusive use by medical staff, and the other is for the passage of patients with indicative symptoms. The layout of the isolation ward was specifically adjusted to accommodate the requirements of patients in single rooms with toilets. Given that the isolation ward is located on the first floor, a dedicated containment-type room with strict protocols for putting on and taking off protective medical attire and equipment was established. Additionally, a dining and resting area with a shower and toilet was established for use by medical staff during rotations in the ophthalmic ward nearby.

The layout of outpatient and emergency facilities followed the principle of "one doctor, one patient per consultation room". Appropriately, positioned signs and warning tape were installed in the pre-examination triage, outpatient registration, diagnosis, and treatment areas. All these measures were implemented to ensure that the distance between each patient waiting in line was more than $1 \mathrm{~m}$.

3. Training, assessment, monitoring, and supervision. Training and lectures pertaining to COVID-19 nosocomial infection control based on the latest specifications, requirements, and relevant standard procedures were administered at the hospital. ${ }^{10}$ The informative value of these measures was assessed via a questionnaire.

In conjunction with the above-described measures, the medical staff departing for Hubei Province to support the national COVID-19 response were trained in occupational health and safety skills, particularly those pertaining to dressing, disassembling, and operating personal protective equipment-which lay the foundation for minimizing the potential for infection in medical staff. Thorough daily clinical front-line inspections were conducted and upscaled to enhance the supervision of the fever 


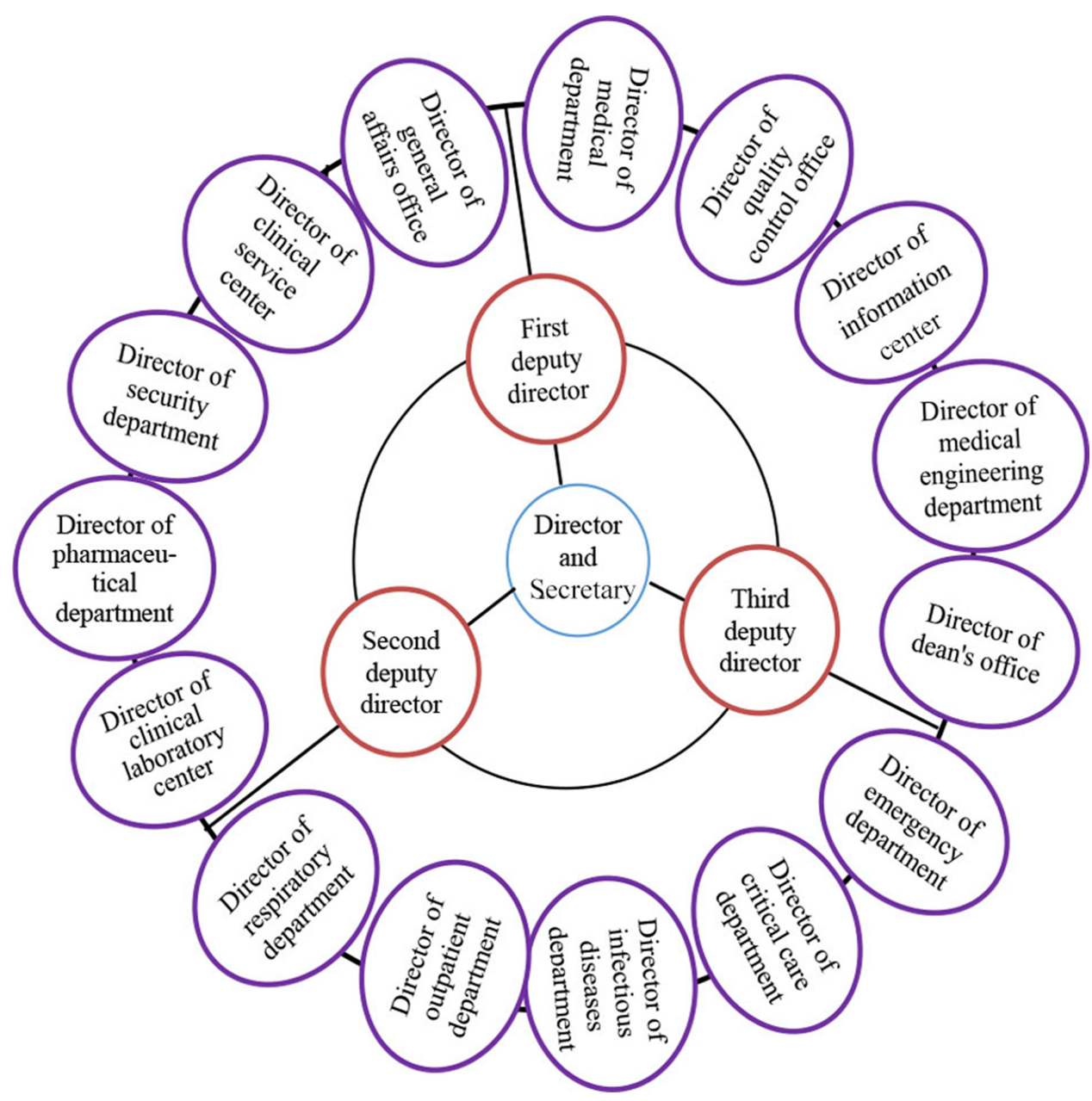

Figure I Central leading organizational structure for prevention and control COVID-19 in the hospital.

outpatient department, outpatient pre-examination triage, respiratory outpatient department, other outpatient departments, emergency pre-examination triage, emergency department, pediatric outpatient and emergency department, isolation ward, intensive care units, infusion room, computed tomography room, temporary storage of medical waste, and laboratories and other key departments. The key elements of this endeavor included personal protection, hand hygiene, disinfection and isolation, patient management, medical waste management, and medical fabric management. Any issues identified via daily inspections and supervision were evaluated and rectified immediately.

4. Preparative assessment and division of patients with different conditions. Medical staff were situated at the entrance of the hospital to check the body temperatures of patients and instruct them to fill in a required form (Figure 2). The main parameters assessed during these screening measures are shown in Table 1.
To prevent cross-infection, the entrance of the hospital has been divided into a patient channel and a medical personnel channel. At the entrance of the outpatient and emergency department, the doctors and nurses on duty are equipped with a pre-examination triage table. Nurses issue these patients wearing masks with air valves, and instruct them how to use medical surgical masks correctly. Under the guidance of medical staff, patients with fever or a relevant contact history were directed to the fever clinic, and other patients were directed to other departments for diagnosis and treatment.

5. Protection and management of medical staff. Medical staff were emphatically reminded that they were required to put on clean gloves after touching blood, body fluids, secretions, excreta, vomit, and objects potentially contaminated by patients, and always wash their hands after taking their gloves off. They were also instructed to wear medical protective masks, goggles, and impervious clothing in situations in which a patient's blood, secretions, or other fluids may 


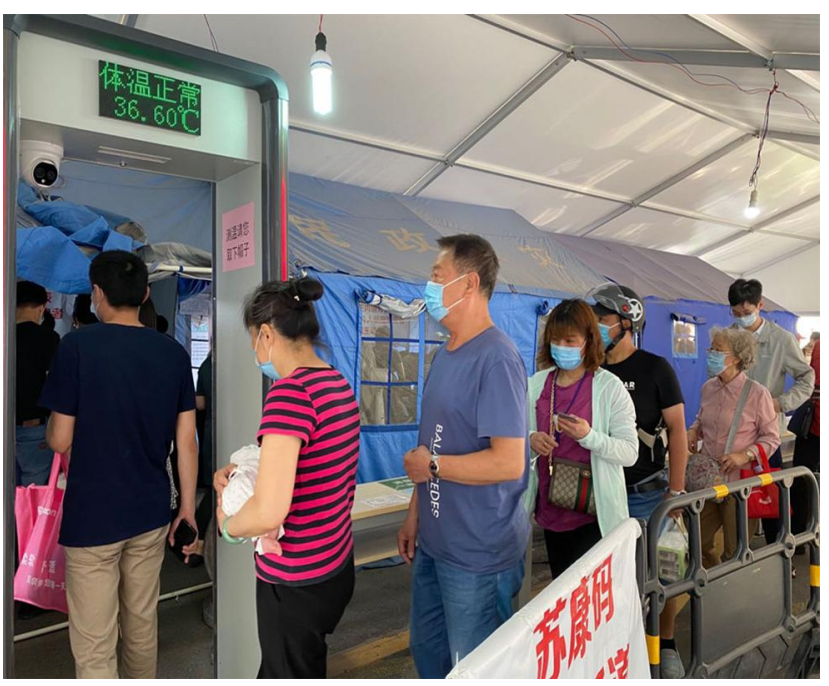

Figure 2 Automatic measurement of body temperature and triage at the entrance of hospital.

splash. During processes such as tracheal intubation, noninvasive ventilation, tracheotomy, cardiopulmonary resuscitation, manual ventilation before intubation, and bronchoscopy in suspected or confirmed COVID-19 patients, many protective methods were implemented. They were minimization of aerosols via air isolation measures, wearing medical protective masks, routine airtightness testing, wearing goggles or a face shield for eye protection, wearing gloves and longsleeved gowns to protect against body fluid penetration, performing procedures in a well-ventilated room, and limiting the number of people in the room to the minimum amount possible while maintaining adequate patient support. ${ }^{12}$

With regard to hand hygiene, the "five moments for hand hygiene" approach was strictly implemented, in accordance with previously reported World Health Organization guidelines. ${ }^{13,14}$ Wearing gloves cannot replace hand hygiene, so staff were reminded of the importance of hand hygiene maintenance after the removal of gloves. It was reiterated to all staff that hand hygiene practices should be conducted before donning protective articles and after each operational step involved in the removal of protective articles, and that hand washing should be conducted thereafter with flowing water. Medical staff were instructed to complete an epidemic prevention and control form every day, including basic information, travel and return time, travel vehicle details, body temperatures, respiratory symptoms, asthenia and/or diarrhea, and other health conditions, and to report to the personnel department.
Table I The Main Screening Contents of the Outpatients and Emergency Patients' Health Declaration

\begin{tabular}{|l}
\hline Outpatients \\
\hline I. Have you been to Hubei or other epidemic areas within 14 days \\
days is the maximum incubation period) ${ }^{5-1}$ '? \\
a. Yes \\
b. No \\
2. Have you contacted with fever patients from Hubei and other \\
epidemic areas, or patients with respiratory symptoms? \\
a. Yes \\
b. No
\end{tabular}

3. Have you recently returned from abroad or in China within 14 days?
a. Yes
b. No

4. During the outbreak (in the past 14 days), have you ever contacted the personnel?

a. Yes

b. No

5. During the epidemic, have you novel coronavirus (nucleic acid positive) infected persons?

a. Yes

b. No

6. Is there an aggregation of disease?

a. Yes

b. No

\section{Emergency patients}

$\mathrm{I}$. Is there a history of travel and residence in Hubei and other epidemic areas within one month?

a. Yes

b. No

2. Is there a suspected exposure history of contacting fever patients or other coronavirus infected pneumonia from Hubei and other epidemic areas?

a. Yes

b. No

3. Is there a history of contact with wild animals?

a. Yes

b. No

4. Is there a gathering of crowd/aggregation incidence?

a. Yes

b. No

6. Patient and ward management. Outpatients were scheduled to attend appointments during staggered periods to reduce patient density. Patients without abnormalities in routine blood tests and chest computed tomography 
admitted to the hospital, and only those with negative results of COVID-19 nucleic acid were to be operated. Given the small number of patients in each ward, it was possible to maintain a bed separation distance of more than $1 \mathrm{~m}$. During hospitalization, one person was allowed to care for a patient, in accordance with the "one escort" policy. When entering or leaving the ward, the accompanying family member was required to provide a registered accompanying card, have his temperature measured, and register at the nurse station. Caregivers were required to undergo epidemiological history screening, thereby they were not replaced without special circumstances. Patients were instructed to wear masks whenever possible, and the accompanying family member was required to wear a mask. Both patients and their accompanying family members were instructed to use appropriate hand hygiene practices and measure their temperature twice a day, and not to congregate, so as to reduce the risk of crossinfection.

7. Management and disinfection of medical waste. The fever clinic and isolation ward were disinfected daily, and the windows of each were open for 30 minutes in the morning and 30 minutes in the evening to facilitate ventilation with fresh air. Where necessary, an air disinfector was used to disinfect the room air. All surfaces and the ground in patient units were wiped and disinfected with chlorine-based disinfectant $(1000 \mathrm{mg} / \mathrm{L})$ twice a day. Small amounts of pollutants were removed carefully using an absorbent material with chlorinebased disinfectant $(2000 \mathrm{mg} / \mathrm{L})$, and then cleaned with a cloth soaked in chlorine-based disinfectant (1000 mg/L). Larger amounts of pollutants were covered with cloth and then sprayed with chlorine-based disinfectant $(2000 \mathrm{mg} / \mathrm{L})$ for 30 minutes prior to removal. ${ }^{15}$ Cleaning tools and disinfection agents reserved for use in different areas were designated with specific marks. After every use, mops and rags were soaked in chlorine-based disinfectant $(1000 \mathrm{mg} / \mathrm{L})$ for 30 minutes, and then cleaned with water before drying.

To the greatest extent possible, disposable diagnostic and treatment equipment was used. Stethoscopes, thermometers, sphygmomanometers, and other medical devices and articles were assigned to a specific person. Reusable devices, appliances, and articles, including electrocardiographs, sphygmomanometers, stethoscopes that could not be specifically assigned to one person, were wiped and disinfected with $75 \%$ ethanol, and those with corrosion-resistant surfaces were wiped and disinfected with chlorine-based disinfectant
(1000 mg/L). Moreover, reusable equipment, utensils, and articles were sealed and marked with "special pathogen" before being transported to the disinfection and supply center.

8. Medical fabric management. Medical fabrics used in the isolation ward including bedspreads, quilt covers, pillow cases, and other fabrics were collected and sealed in a closed soluble medical fabric packaging bag (orange and red) labeled with "special pathogen" at the bedside. The washing company first soaked these special bags in chlorine-based disinfectant $(500 \mathrm{mg} / \mathrm{L})$ for half an hour, then washed and disinfected them in accordance with their normal cleaning process. During the whole process, transport personnel wore disposable hats, surgical masks, latex gloves, and work clothes as well as implementing appropriate hand hygiene practices. The specific personnel collected the medical waste produced in the fever clinic and isolation ward in special buckets, stored at designated points, and handed to a professional company by special transport vehicle.

\section{Results}

Since the first COVID-19 case was reported outside Wuhan, our hospital started the detection of SARS-CoV-2 and treatment of cases of COVID-19 infection. Between 21 January 2020 and 27 February 2020 the fever clinic at our hospital received 995 patients with fever and confirmed 3 cases of COVID-19 infection after eliminating 6 suspected cases.

Thereafter, more than 1860 patients with fever or a relevant contact history visited our hospital (Update to 28 May 2020), there were no new cases of COVID-19 infection, no cases of medical staff infection, and no COVID-19 related deaths at our hospital after the implementation of the infection prevention and control strategies.

\section{Discussion}

Since 21 January 2020, our hospital has been one of the designated hospitals for the referral of suspected COVID-19 cases in Jiangsu Province, China. After the initial reports of infections and outcomes in Wuhan Medical Staff, our hospital actively implemented a series of infection control measures to prevent nosocomial spread of COVID-19. Since the epidemic was declared, most countries have applied strict bans on flights from China. Conversely, other countries have implemented border screening measures such as temperature checks. ${ }^{5}$ Various measures have been adopted since the COVID-19 outbreak, of which social isolation and lockdown play important roles in preventing its spread. ${ }^{16}$ There is no doubt that control strategies including school closure and social distancing have considerably reduced the total number of cases. 
Given the impossibility of social isolation and lockdown of patients, the hospital applied the principle of "one doctor, one patient per consultation room" and a "one escort policy" to reduce the population density, thereby reducing the chance of viral infection.

As we know, the viruses can be completely inactivated at high temperatures. ${ }^{17}$ A growing number of recent studies suggest that the spread of SARS-CoV-2 and its case fatality rate may vary in regions with different climates and temperatures. $^{18,19}$ In the 10 most affected provinces in China, empirical results obtained from 22 January 2020 to 31 March 2020 indicate that there was a positive relationship between temperature and COVID-19 in Hubei, Hunan, and Anhui, and a negative relationship in the provinces of Zhejiang and Shandong, whereas in the remaining five provinces including Jiangsu there were mixed trends. ${ }^{20}$ All these conflicting data may be explained by differences in the numbers of COVID-19 cases, temperature, and hospital facilities. Huang et $\mathrm{al}^{6}$ recently reported that $60.0 \%$ of confirmed cases of COVID-19 occurred in places where the air temperature ranged from $5.0^{\circ} \mathrm{C}$ to $15.0^{\circ} \mathrm{C}$, with a peak in cases at $11.5^{\circ} \mathrm{C}$. Accordingly, densely populated areas such as hospitals can be advised to control temperature ranges to reduce the probability of cross-infection.

Public health education is one of the effective measures for controlling SARS-COV-2, as it is for controlling other transmissible diseases. ${ }^{21}$ At our hospital, all staff engage in COVID-19 control and prevention programs, and learn about laboratory biosafety, prevention and control, and technical guidelines via media and online services. As a result, each staff member knows about COVID-19 transmission, and the importance of transparent data flow and up-to-date reports of confirmed COVID-19 cases and deaths. Such things are critical for preventing and controlling the spread of COVID-19.

Certainly, we should further enhance awareness and the ability of medical staff to prevent and control COVID19 , optimize the control measures and processes of medical institutions, and improve social security strategies. We should also strengthen the supervision and inspection of classified collection, storage, and transport of medical waste to avoid its accumulation, and strive to create a healthy environment. While the above-described COVID-19 prevention and control strategies were in place, the fever clinic at our hospital received 995 patients with fever and confirmed 3 cases of COVID-19 infection after eliminating 6 suspected cases as of 27 February 2020. Interestingly, there were no cases of medical staff infection and no COVID-19 related deaths at our hospital after the implementation of the infection prevention and control strategies. Notably, the spread of COVID-19 is affected by several factors. We hope that more measures will be introduced to allow medical staff to take better care of themselves so that they can combat SARS-CoV-2 without compromising their personal wellbeing.

\section{Conclusion}

The current study provides a protocol that could be used in severe epidemics by relevant medical departments in similar cities worldwide.

\section{Author Contributions}

Both authors made significant contributions to the work reported, whether that was in the conception and design of the study, its execution, data acquisition, analysis, and interpretation, or in all these areas; took part in drafting, revising, or critically reviewing the manuscript; approved of the version to be published; have agreed on the journal to which the article has been submitted; and agreed to be accountable for all aspects of the work.

\section{Disclosure}

The authors declare that they have no competing interests for this work.

\section{References}

1. Lu R, Zhao X, Li J, et al. Genomic characterization and epidemiology of 2019 novel coronavirus: implications of virus origins and receptor binding. Lancet. 2020;395(10224):565-574. doi:10.1016/S01406736(20)30251-8

2. Wu JT, Leung K, Leung GM. Nowcasting and forecasting the potential domestic and international spread of the 2019-nCoV outbreak originating in Wuhan, China: a modelling study. Lancet. 2020;395:689-697. doi:10.1016/S0140-6736(20)30260-9

3. Qiu Y, Chen X, Shi W. Impacts of social and economic factors on the transmission of coronavirus disease 2019 (COVID-19) in China. J Popul Econ. 2020;33:1127-1172. doi:10.1007/s00148-020-00778-2

4. Leung K, Wu JT, Liu D, Leung GM. First-wave COVID-19 transmissibility and severity in China outside Hubei after control measures, and second-wave scenario planning: a modelling impact assessment. Lancet. 2020;395(10233):1382-1393. doi:10.1016/S0140-6736(20) 30746-7

5. WHO. Coronavirus disease (COVID-2019) situation reports. Available from: https://www.who.int/emergencies/diseases/novelcoronavirus2019/situation-reports/. Accessed March 29, 2020.

6. Huang ZW, Huang JP, Gu QQ, Du PY, Liang HB, Dong Q. Optimal temperature zone for the dispersal of COVID-19. Sci Total Environ. 2020;736:139487. doi:10.1016/j.scitotenv.2020.139487

7. Piontti PY, Mu K, Rossi L, et al. The effect of travel restrictions on the spread of the 2019 novel coronavirus (COVID-19) outbreak. Science (New York). 2020;9757:1-12. doi:10.1126/science.aba9757 
8. Li R, Pei S, Chen B, et al. Substantial undocumented infection facilitates the rapid dissemination of novel coronavirus (SARS-CoV2). Science (New York). 2020;3221:1-9. doi:10.1126/science.abb3221

9. Jocelyne B, Campbell WS, Terry M. COVID-19: infection prevention and control guidance for all ultrasound practitioners. Aust J Ultrasound Med. 2020;23. doi:10.1002/ajum.12210.

10. Yang M, Wang HM, Zhi L, et al. Prevention and control of COVID-19 infection in a Chinese mental health center. Front Med. 2020;7:356. doi:10.3389/fmed.2020.00356

11. Ayenigbara IO. COVID-19: an international public health concern. Cent Asian J Glob Health. 2020;9(1):11. doi:10.5195/cajgh.2020.466

12. Healthcare Workers. Clinical care guidance for healthcare professionals about coronavirus (COVID-19). National Center for Immunization and Respiratory Diseases (NCIRD), Division of Viral Diseases 2020. Available from: www.cdc.gov/coronavirus/2019nCoV/hcp/index.html.

13. Pittet D, Donaldson L. Clean care is safer care: the first global challenge of the WHO World Alliance for patient safety. Am $J$ Infect Control. 2005;33(8):476-479. doi:10.1016/j.ajic.2005.08.001

14. Biswal M, Angrup A, Rajpoot S, et al. Hand hygiene compliance of patients' family members in India: importance of educating the unofficial 'fourth category' of healthcare personnel. J Hosp Infect. 2020;104(4):425-429. doi:10.1016/j.jhin.2019.09.013

15. Yang Y, Wang HL, Chen K, Zhou J, Deng SP, Wang Y. Shelter hospital mode: how to prevent novel coronavirus infection 2019 (COVID-19) hospital-acquired infection? Infect Control Hosp Epidemiol. 2020;41:1-4. doi:10.1017/ice.2020.97
16. Tomar A, Gupta N. Prediction for the spread of COVID-19 in India and effectiveness of preventive measures. Sci Total Environ. 2020;728:138762. doi:10.1016/j.scitotenv.2020.138762

17. Park JE, Son WS, Ryu Y, Choi SB, Kwon O, Ahn I. Effects of temperature, humidity, and diurnal temperature range on influenza incidence in a temperate region. Influenza Other Respir Viruses. 2020;14(1):11-18. doi:10.1111/irv.12682

18. Wang LS, Li J, Guo SM, et al. Real-time estimation and prediction of mortality caused by COVID-19 with patient information based algorithm. Sci Total Environ. 2020;727:138394. doi:10.1016/j. scitotenv.2020.138394

19. Prata DN, Rodrigues W, Bermejo PH. Temperature significantly changes COVID-19 transmission in (sub) tropical cities of Brazil. Sci Total Environ. 2020;729:138862. doi:10.1016/j. scitotenv.2020.138862

20. Farrukh S, Umer S, Zeeshan F, Najaf I, Shujahat H, Fayyaz A. Asymmetric nexus between temperature and COVID-19 in the top ten affected provinces of China: A current application of quantile-onquantile approach. Sci Total Environ. 2020;736:139115. doi:10.1016/ j.scitotenv.2020.139115

21. Majid N, Hamid RN. COVID-19 outbreak: application of multi-gene genetic programming to country-based prediction models. Electron J Gen Med. 2020;17:em247. doi:10.29333/ejgm/8232
Risk Management and Healthcare Policy

\section{Publish your work in this journal}

Risk Management and Healthcare Policy is an international, peerreviewed, open access journal focusing on all aspects of public health, policy, and preventative measures to promote good health and improve morbidity and mortality in the population. The journal welcomes submitted papers covering original research, basic science, clinical \& epidemiological studies, reviews and evaluations, guidelines, expert opinion and commentary, case reports and extended reports. The manuscript management system is completely online and includes a very quick and fair peer-review system, which is all easy to use. Visit http://www.dovepress.com/testimonials.php to read real quotes from published authors. 